\title{
CDCA1/2/3/5/7/8 as novel prognostic biomarkers and CDCA4/6 as potential targets for gastric cancer
}

\author{
Zhaoxing Li ${ }^{1}$, Zhao Liu ${ }^{2}$, Chuang $\mathrm{Li}^{3}$, Qingwei Liu ${ }^{1}$, Bibo Tan ${ }^{1}$, Yu Liu ${ }^{1}$, Yifei Zhang ${ }^{4}$, Yong Li $^{1}$ \\ ${ }^{1}$ Department of General Surgery, The Fourth Affiliated Hospital of Hebei Medical University, Shijiazhuang, China; ${ }^{2}$ Key Laboratory of \\ Carcinogenesis and Translational Research (Ministry of Education), Department of Gastrointestinal Surgery, Peking University Cancer Hospital and \\ Institute, Beijing, China; ${ }^{3}$ The Second Hospital of Shijiazhuang, Shijiazhuang, China; ${ }^{4}$ Hebei General Hospital, Shijiazhuang, China \\ Contributions: (I) Conception and design: Z Li, Z Liu; (II) Administrative support: Y Li; (III) Provision of study materials or patients: Z Li; \\ (IV) Collection and assembly of data: Z Li; (V) Data analysis and interpretation: Z Li, Z Liu; (VI) Manuscript writing: All authors; (VII) Final \\ approval of manuscript: All authors. \\ Correspondence to: Yong Li. The Fourth Affiliated Hospital of Hebei Medical University, 12 Jiankang Road, Shijiazhuang 050019, China. \\ Email: yongli_hbfh@163.com.
}

Background: Increasing evidence had suggested that cell division cycle-associated (CDCA) family proteins play prominent roles in multiple types of cancer. However, the expression pattern and prognostic value of CDCAs in gastric cancer were still poorly understood.

Methods: In this study, bioinformatics was used for the first time to comprehensively discuss the expression changes of the CDCA protein family in gastric cancer. We studied the transcription and survival data of CDCAs in patients with gastric cancer in Oncomine, GEPIA, DAVID, cBioportal, and other databases.

Results: We identified that the CDCA 1/2/3/4/5/6/7/8 were overexpressed gastric cancer than in normal tissues. There was no significant difference in CDCAs expression among different gastric cancer stages. High expression of CDCA4/6 in patients with gastric cancer was closely related to low overall survival (OS), first progression survival (FPS), and post-progression survival (PPS). In contrast, high CDCA1/2/3/5/7/8 expression predicted a better prognosis. The genetic mutation rate of $C D C A 2$ and $C D C A 4$ was $4 \%$, ranking first. The main biological process of CDCAs protein family enrichment was cell division, the main cell component involved was centromeres of chromosomes, and the main molecular function involved was protein binding.

Conclusions: The study suggested that CDCA1/2/3/5/7/8 were expected to be new prognostic markers for gastric cancer, and CDCA4/6 might be potential targets for the treatment of gastric cancer.

Keywords: Cell division cycle-associated proteins (CDCA proteins); gastric cancer; bioinformatics; prognosis

Submitted Feb 16, 2021. Accepted for publication May 10, 2021.

doi: $10.21037 /$ tcr-20-1050

View this article at: https://dx.doi.org/10.21037/tcr-20-1050

\section{Introduction}

Gastric cancer was one of the most significant causes of cancer-related morbidity and mortality throughout the world. The incidence of gastric cancer in East Asia, particularly China, increases strikingly, which placed a huge burden on global public health (1). Although treatments such as surgery and chemotherapy had improved the survival and prognosis, the 5 -year survival rate remained unsatisfactory. Therefore, the identification of novel prognostic biomarkers and therapeutic targets related to the carcinogenesis of gastric cancer was of great value.

Cell division cycle-associated (CDCA) protein family acted as crucial regulators in the process of cell division and proliferation. Increasing evidence had suggested that CDCAs played prominent roles in multiple types of cancer, 
such as breast cancer, lung cancer, colorectal cancer, bladder cancer, oral cancer, and so on (2-6). For instance, the study by Thang et al. demonstrated that CDCA1 may represent a new prognostic and therapeutic target for oral cancer (2). The findings of Adams et al. had highlighted CDCA3 as a prognostic factor in lung cancer (7). Moreover, some studies had proposed that CDCA3 might serve as an oncogene in gastric cancer (GC) (8). However, the expression pattern of CDCA protein subtypes in gastric cancer was not clear yet. Meanwhile, due to a large number of CDCA protein subtypes, the key subtype had not been preliminarily screened for the study of gastric cancer.

In the present study, we utilized bioinformatics analysis to study the expression differences and prognostic value of CDCA subtypes in gastric cancer for the first time. Initially, we validated the expression of the CDCA protein family in various malignant tumors in several databases. Subsequently, we identified the prognostic value, genetic alterations, and gene ontology analysis of key CDCA members in gastric cancer tissues. We present the following article in accordance with the MDAR checklist (available at https://dx.doi.org/10.21037/tcr-20-1050).

\section{Methods}

\section{Oncomine analysis}

The Oncomine (http://www.oncomine.org) database was a publicly accessible online database, which integrated numerous published tumor microarray data (9). We used this online database to identify the differences in the expression of major CDCA subtypes between different tumor tissues and normal tissues. Subsequently, we focused on verifying the expression of the main CDCA protein subtypes in gastric cancer datasets. The mRNA expression of CDCA protein between cancer and normal tissues was compared by $t$-test. The $\mathrm{P}$ value was set up at 0.05 and the $\log 2$ (fold change) was defined as 1.5 .

\section{GEPIA analysis}

Gene Expression Profiling Interactive Analysis (GEPIA, http://gepia.cancer-pku.cn), a novel interactive online tool, was applied to conduct various functions using the sequencing expression data of cancer samples from TCGA and GTEx projects, such as tumor/normal differential expression analysis, pathological stage analysis, and so on (10). We further verified major CDCA subtypes expression level in gastric cancer using GEPIA, and screened out the differentially expressed genes consistent with Oncomine database results. Meanwhile, with the aid of GEPIA, we explored whether there are differences in the expression of these genes in different pathological stages.

\section{Survival analysis}

Kaplan-Meier plotter (http://kmplot.com/analysis/), which collected a large amount of gene chip data and clinical data from EGA, GEO, and TCGA databases, could assess the survival impact of more than 50,000 genes on 21 sorts of cancers (11). The prognostic values of the 8 selected genes were assessed by calculating the overall survival (OS), first progression survival (FP), and post-progression survival (PPS).

\section{Genetic alteration analysis}

The cBioPortal for Cancer Genomics (https://www. cbioportal.org/) was an open comprehensive platform, which integrated data mining, data integration, and visualization (12). We selected the gastric adenocarcinoma dataset (TCGA, Provisional) in the TCGA database and utilized cBioportal to analyze the genetic variation of hub genes.

\section{Enrichment analysis and protein-protein interaction (PPI) analysis}

To clarify potential biological meanings behind the hub genes, we utilized DAVID (the Database for Annotation, Visualization and Integrated Discovery, version 6.8) to carry out the GO (Gene Ontology) enrichment analysis and KEGG (Kyoto Encyclopedia of Genes and Genomes) pathway enrichment analysis (13). The $\mathrm{P}$ value cutoff was set to 0.05 and the result was visualized through the ggplot package of $\mathrm{R}$ software (version 3.5.3). Through the enrichment analysis in the DAVID database, we used the KEGG database (https://www.kegg.jp/) to identify the most promising signaling pathway related to the hub genes. The protein-protein interaction network was then constructed using the STRING (Search Tool for the Retrieval of Interacting Genes, https://string-db. org/) database to identify the interaction relationship and adjacent network among genes (14). The study was conducted in accordance with the Declaration of Helsinki 

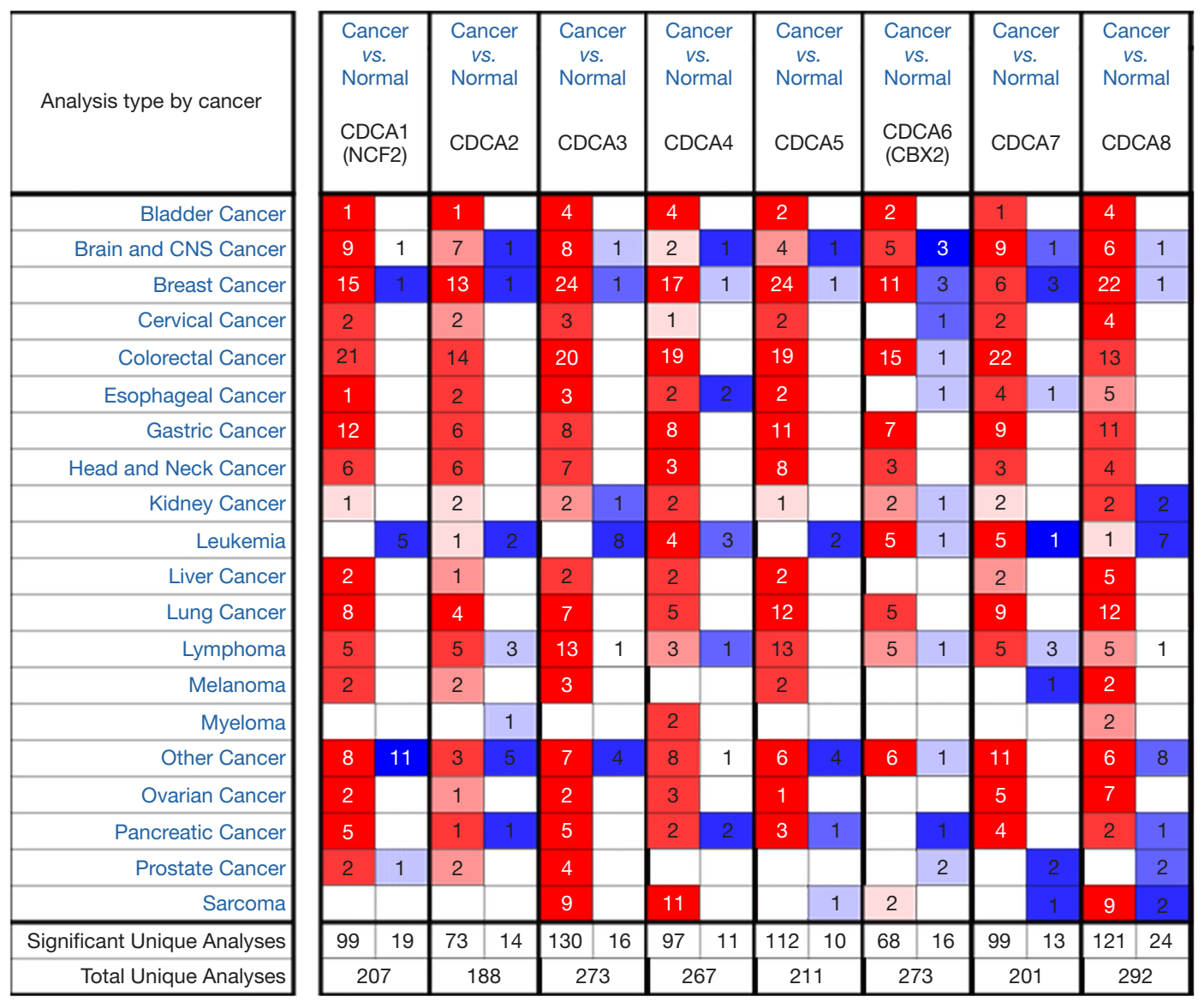

Figure 1 The mRNA expression of CDCA protein family in different cancers (Oncomine database). The figure showed the numbers of datasets with statistically significant mRNA over-expression (red) or down-expression (blue) of related gene. The $\mathrm{P}$ value was set up at 0.05 . The number in each cell represented the number of analyses that met the threshold. We utilized the percentile of related gene in the top of all genes measured in each research to analyze the gene rank. Cell color was determined by the best gene rank percentile for the analyses within the cell.

(as revised in 2013).

\section{Statistical analysis}

The difference of CDCAs mRNA expression between cancer tissues and adjacent normal tissues was compared by $t$-test. The $\mathrm{P}$ value was set to 0.05 , and $\log 2$ (fold change) was defined as 1.5. Kaplan-Meier test and Log-rank test were used to compare the difference of survival prognosis among different gene expression levels. $\mathrm{P}<0.05$ was considered statistically significant. The biological processes, cellular components and molecular functions of key genes were analyzed by GO enrichment analysis.

\section{Results}

\section{Expression level of CDCAs in gastric cancer}

We utilized the Oncomine database to preliminarily analyze the expression of the main subtypes of CDCAs in 20 malignant tumors. The results revealed that the main CDCA subtypes-CDCA1, CDCA2, CDCA3, CDCA4, CDCA5, CDCA6, CDCA7, CDCA8 - expressed differently in various types of tumors compared with the corresponding normal tissues (Figure 1). We further analyzed the expression of CDCAs in gastric cancer using the Oncomine database. The results showed that the expression of CDCA1, CDCA2, CDCA3, CDCA4, CDCA5, CDCA6, 
Table 1 Significant difference in expression of CDCA protein family subtypes between gastric cancer and normal gastric tissues

\begin{tabular}{|c|c|c|c|c|}
\hline CDCA subtype & Types of gastric cancer vs. normal & Fold change & $P$ value & $\mathrm{t}$ value \\
\hline CDCA2 & Gastric cancer vs. normal & 2.774 & $1.29 \mathrm{E}-5$ & 4.336 \\
\hline CDCA3 & Gastric cancer vs. normal & 2.106 & $2.19 \mathrm{E}-5$ & 4.575 \\
\hline CDCA4 & Gastric intestinal type adenocarcinoma vs. normal & 2.214 & $7.44 \mathrm{E}-12$ & 8.857 \\
\hline CDCA6 & Diffuse gastric adenocarcinoma vs. normal & 2.290 & $6.01 \mathrm{E}-9$ & 6.862 \\
\hline CDCA7 & Gastric cancer vs. normal & 2.111 & 7.94E-8 & 5.490 \\
\hline CDCA8 & Gastric intestinal type adenocarcinoma vs. normal & 2.028 & $5.28 \mathrm{E}-10$ & 7.560 \\
\hline
\end{tabular}

CDCA7, and CDCA8 was up-regulated in gastric cancer tissues with statistical significance. In the meantime, the fold changes of CDCA1, CDCA2, CDCA3, CDCA4, CDCA5, CDCA6, CDCA7 and CDCA8 were 2.700, 2.774, 2.106, 2.214, 2.674, 2.290, 2.111 and 2.028, respectively (Table 1).

\section{The relationship between CDCAs expression level and clinical stages in patients with gastric cancer}

GEPIA (Gene Expression Profiling Interactive Analysis) was applied to verify the expression level of claudins. A total of 408 gastric cancer samples and 211 normal gastric tissues were included in the study dataset. The expression level of CDCA1, CDCA2, CDCA3, CDCA4, CDCA5, CDCA6, CDCA7, and CDCA8 in gastric cancer tissues was higher than that in normal tissues (Figure 2). Also, we found that although CDCA1, CDCA2, CDCA3, CDCA4, CDCA5, CDCA6, CDCA7, and CDCA8 were significantly different between gastric cancer tissues and normal samples, there was no clear difference in various stages of gastric cancer (Figure 3).

\section{Increased expression of CDCA1/2/3/5/7/8 and decreased expression of CDCA4/6 are associated with improved prognosis of gastric cancer}

We further analyzed the relationship between the hub genes-CDCA1, CDCA2, CDCA3, CDCA4, CDCA5, $C D C A 6, C D C A 7, C D C A 8$ - and the prognosis and survival of gastric cancer. Kaplan-Meier plotter was applied to plot the relationship between gene expression level and OS, FPS, and PPS of gastric cancer. The results showed that the higher expression levels of CDCA1, CDCA2, CDCA3,
CDCA5, CDCA7 in gastric cancer patients indicated worse OS, FP, PPS. On the contrary, gastric cancer patients with high expression level of CDCA4, CDCA6 showed better prognosis and survival (Figures 4,5). The above data differences had statistical significance. Notably, lower expression of CDCA8 predicted better OS and PPS, but there was no significant difference in FP (Figure 5).

\section{Genetic alteration analysis and PPI construction of CDCAs}

We utilized cBioportal to analyze the distribution of genetic alterations of the hub genes in gastric cancer samples. The results showed that among the 8 hub genes, the mutation rate of $C D C A 2$ and $C D C A 1$ (NUF2) genes was $4 \%$, ranking first. The gene mutation rates of $C D C A 3$ and $C D C A 5$ were the lowest, at only $1.5 \%$. The cumulative genetic alteration rate of each subtype of gastric cancer ranged from $10 \%$ to $30 \%$ (Figure 6). We constructed the PPI network and showed the connections among the hub genes, and found some adjacent genes-BUB1, SPC25, AURKB, NDC80, SPC24.

\section{GO and KEGG enrichment analysis of bub CDCAs}

We used GO and KEGG enrichment analysis to attempt to expound the biological meaning behind hub genes. GO analysis mainly referred to three sections: biological process, cellular component and molecular function. When it came to biological process (BP), they were mainly related to cell division, sister chromatid cohesion, mitotic nuclear division, and so on. As for cellular component, the hub genes primarily participated in nucleus, nucleoplasm, chromosome. Concerning molecular function, they were 
CDCA1

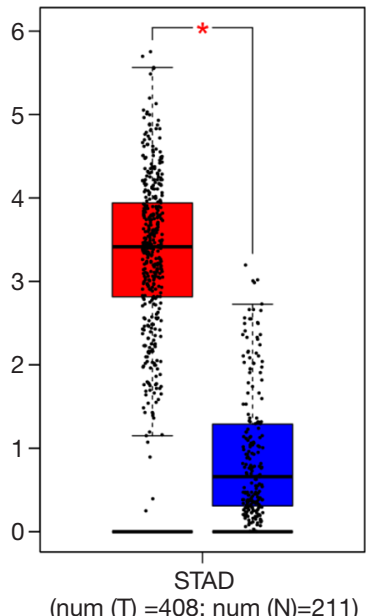

(num $(T)=408 ;$ num $(N)=211)$
CDCA2

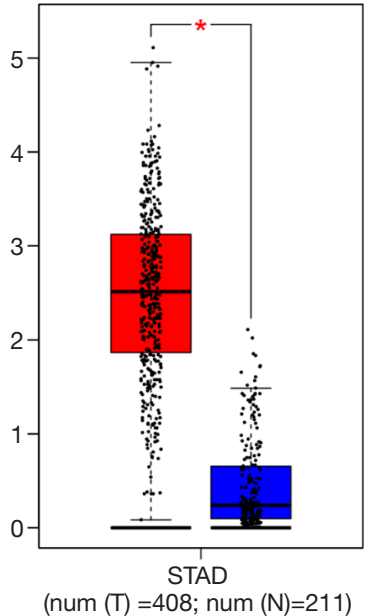

CDCA3

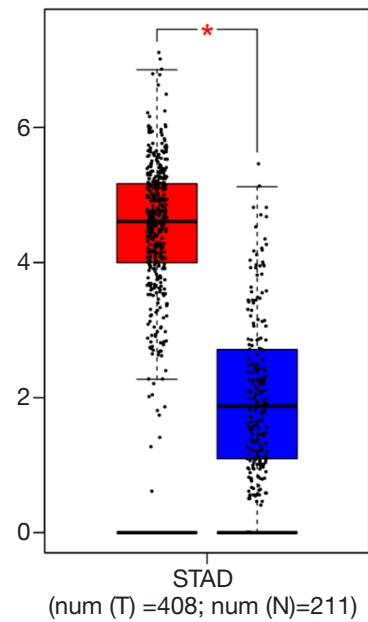

CDCA4

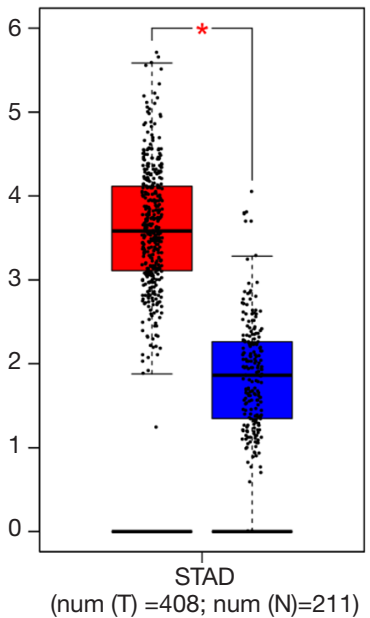

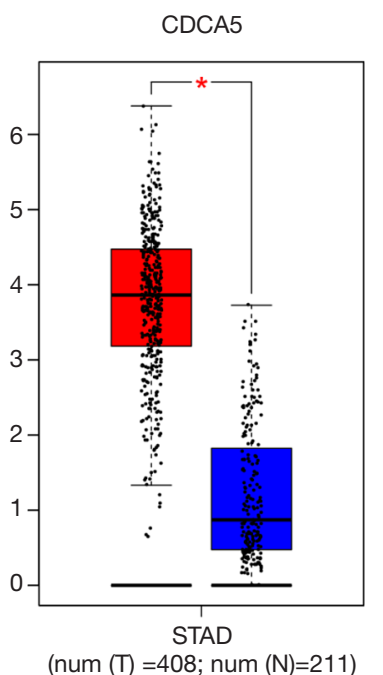
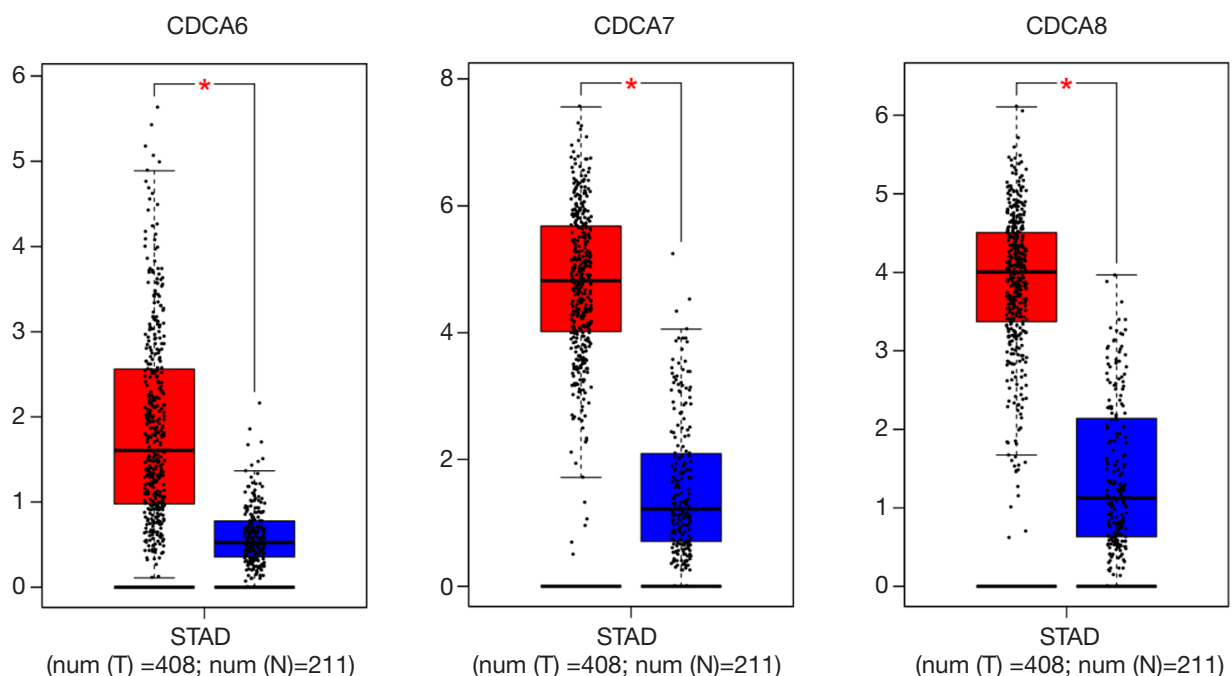

Figure 2 The mRNA expression level of CDCAs in gastric cancer (GEPIA). There were 480 gastric cancer samples, represented by red color. The blue color represented the 211 adjacent normal gastric tissues.

significantly enriched in structural molecule activity, identical protein binding. Besides, variations in KEGG pathway enrichment analysis were closely associated to protein binding (Figure 7). KEGG enrichment analysis of these hub genes showed no significant enrichment pathway.

\section{Conclusions}

In the present study, we used bioinformatics analysis to preliminarily identify the expression patterns of CDCA subtypes in different tumor samples and corresponding normal tissues for the first time. The results revealed that there were significant differences in the expression of different protein subtypes in different tumor tissues, suggesting that CDCAs were closely associated with the occurrence and development of malignant tumors. The roles of CDCAs in the tumorigenesis of gastric cancer were largely unknown. In this paper, we determined the prognostic value and expression patterns of key CDCA subtypes through a comprehensive analysis of multiple databases. These pointed out the key direction for further studying the relationship between CDCAs and gastric cancer in the future.

CDCA1, also known as NUF2, coded for a protein that 

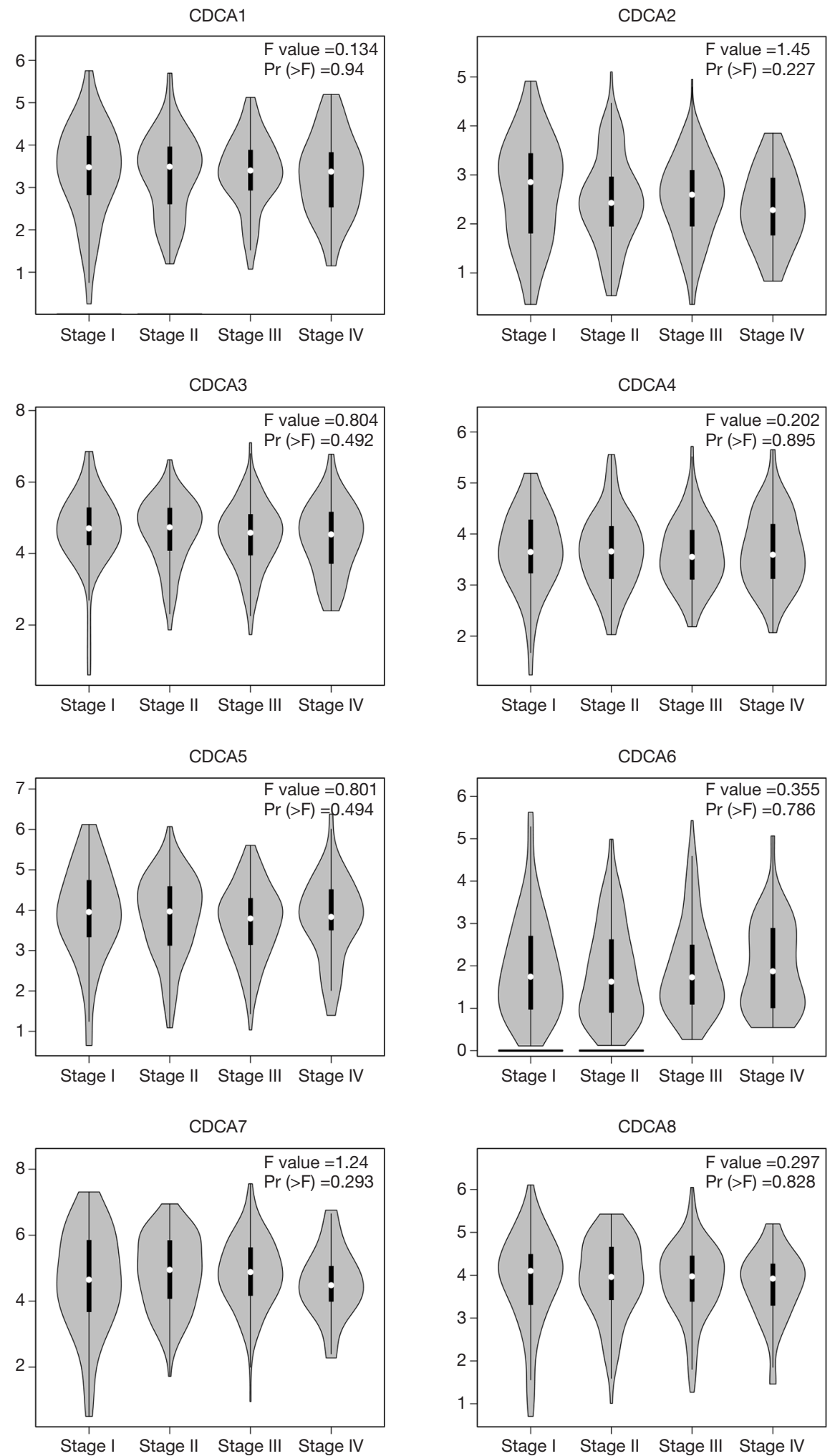

Figure 3 Correlation between CDCAs expression level and tumor stage in gastric cancer patients (GEPIA). 

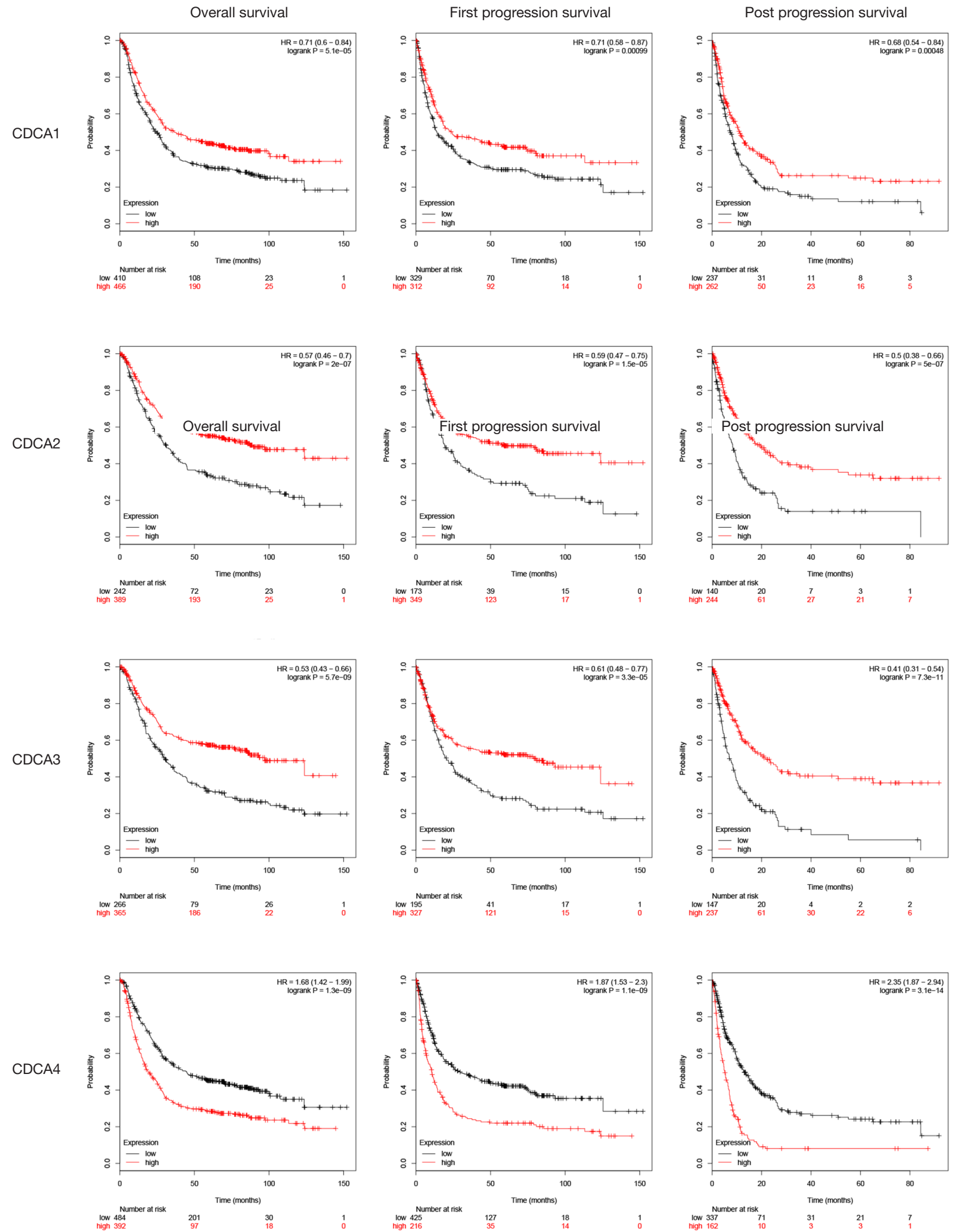

Figure 4 The prognostic value of CDCA1/2/3/4 in gastric cancer patients (Kaplan-Meier plotter). 

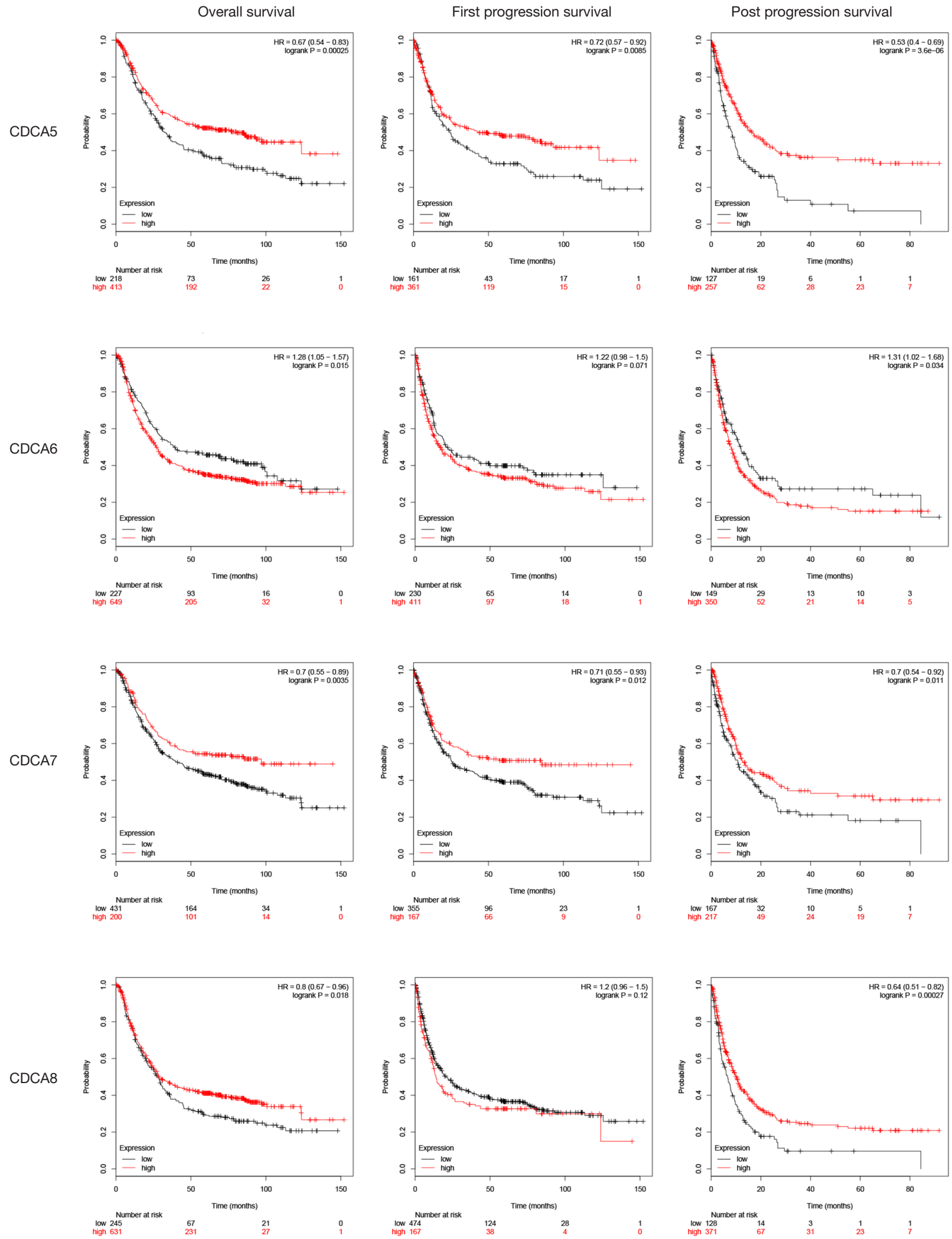

Figure 5 The prognostic value of CDCA5/6/7/8 in gastric cancer patients (Kaplan-Meier plotter). 
A

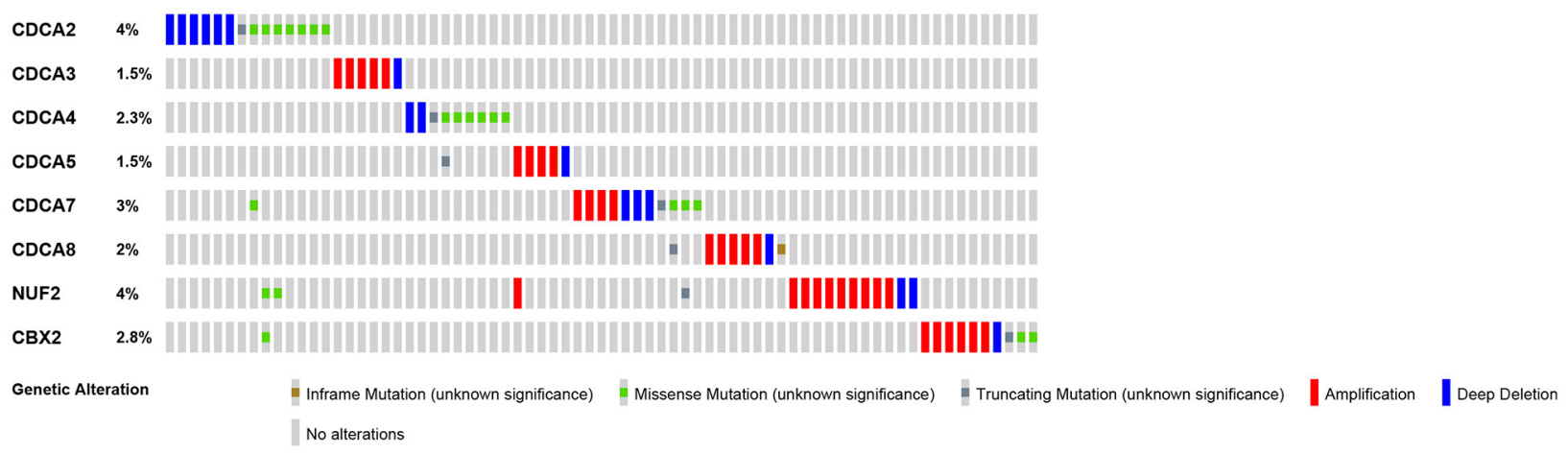

B

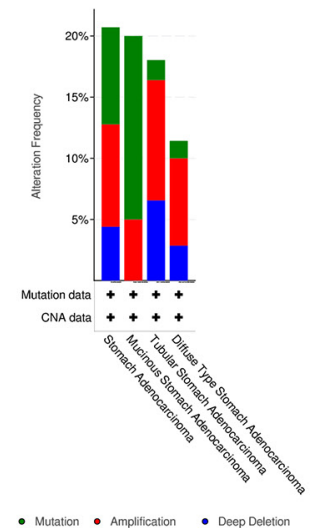

C

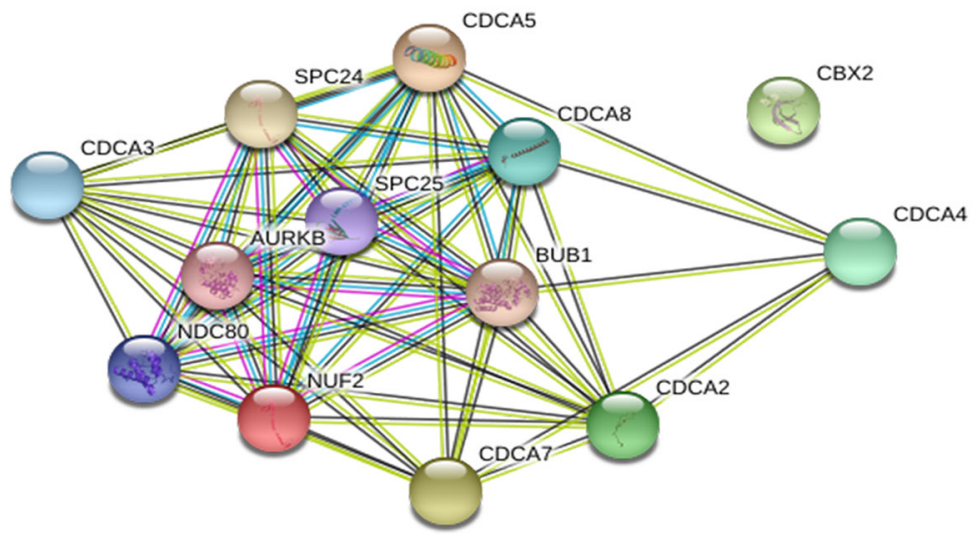

Figure 6 The mutation analysis and the protein-protein interaction (PPI) network construction of CDCAs in gastric cancer. (A) The genetic alteration proportion and distribution of CDCAs in gastric cancer (cBioportal). (B) The alteration frequency distribution of CDCAs in gastric cancer subtypes (cBioportal). (C) The PPI network construction of CDCA protein family.

was critical for nuclear division and microtubule stability and played a regulatory role in chromosome separation (15). CDCA1 knockout might result in the death of mitotic cells. CDCA1 had been reported to be upregulated in multiple types of cancer, such as oral cavity carcinoma (OCC), lung cancer (LC), colorectal cancer CRC, prostate cancer (PC), and even gastric cancer (GC) $(2-4,16,17)$. Studies indicated that high CDCA1 expression was associated with poor prognosis in oral cancer patients and colorectal cancer patients, and knockdown of CDCA1 might significantly induce apoptosis of OCC cells, CRC cells, and PC cells $(2,4,17)$. Obara conducted a phase I clinical trial for patients with castration-resistant prostate cancer using a CDCA1 peptide vaccination, which suggested that CDCA1-derived peptide vaccine might effectively induce peptide-specific cytotoxic $\mathrm{T}$ lymphocyte (CTL) responses and played a role in cancer inhibition (18). In this study, the data demonstrated that CDCA1 was overexpressed in gastric cancer compared with normal gastric tissues, which was consistent with Ohnuma's findings (16). Notably, our data showed that high CDCA1 expression was associated with better OS, FP, PPS.

CDCA2 was a nuclear protein, which was considered to participate in the regulation of DNA damage response in the cell cycle (19). Uchida et al. demonstrated that CDCA2 expression level was upregulated in oral squamous cell carcinoma (OSCC), and CDCA2 might be related to OSCC progression by preventing cell-cycle arrest and apoptosis (20). The study by Shi et al. revealed that CDCA2 was upregulated in lung adenocarcinoma (LAC) compared with normal lung tissues, and the overexpression of CDCA2 might promote the proliferation of LAC cells by upregulating CCNE1. And CCDA2 might act as an independent risk factor for OS in LAC patients (21). Feng et al.'s findings showed that overexpression of CDCA2 might promote CRC cell proliferation through activation 
A

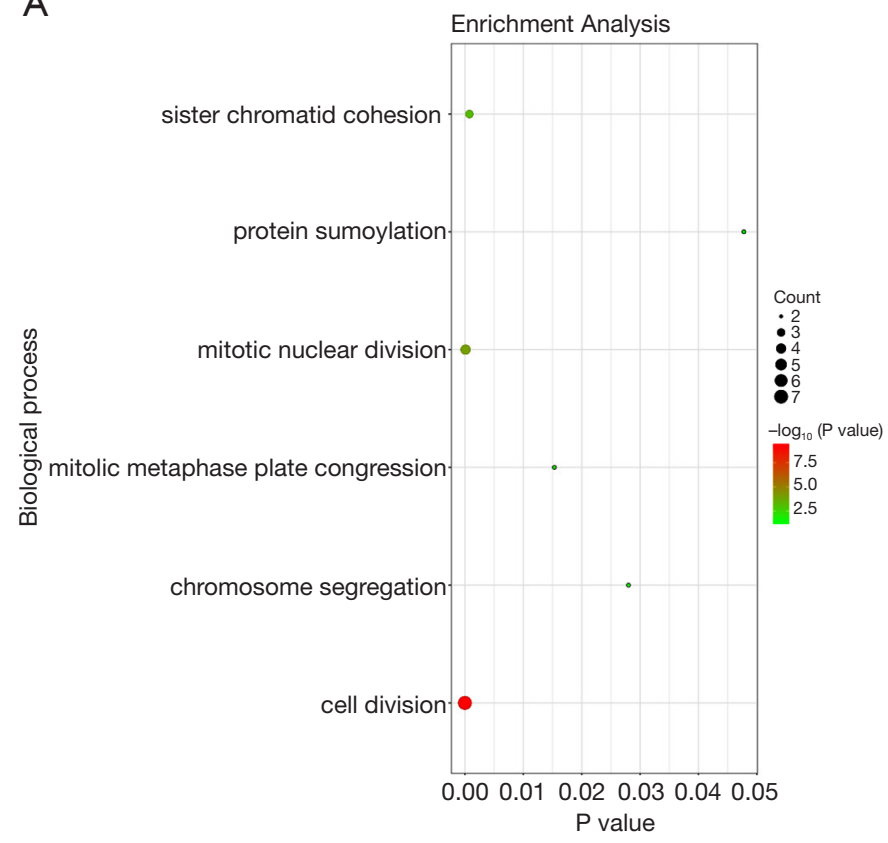

C

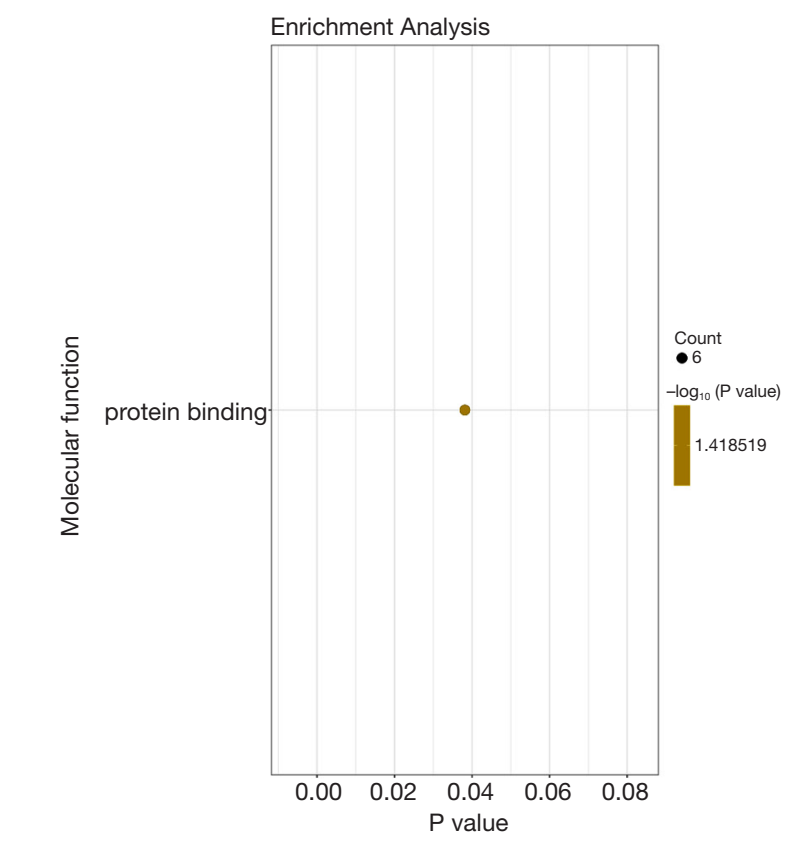

B

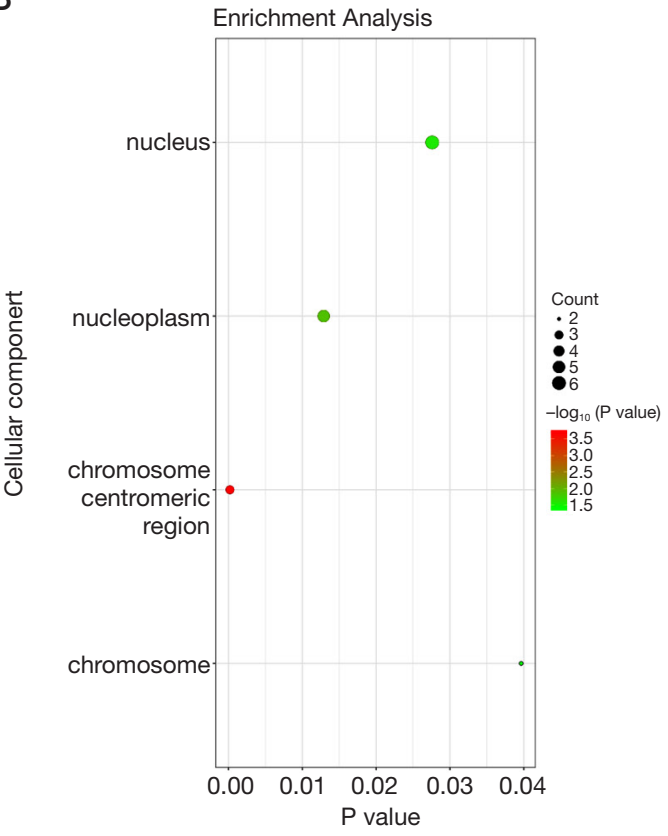

Figure 7 The Gene Ontology (GP) enrichment analysis of CDCAs using DAVID. The GO enrichment analysis mainly contained three terms, including biological process (A), cellular components (B), molecular functions (C).

of the PI3K/AKT pathway (22). Our data showed that the expression of CDCA2 was increased in gastric cancer, and elevated CDCA2 expression predicted better OS, FP, PPS in gastric cancer patients.

CDCA3 was commonly thought to be associated with night blindness. In recent years, it had been found that the expression level of CDCA3 in breast cancer, non-small cell lung cancer, oral squamous cell carcinoma, gastric cancer, and colorectal cancer was increased compared with the normal adjacent tissues (7,8,23-25). Studies also found that 
its high expression was associated with poor prognosis in patients with breast cancer and non-small cell lung cancer $(7,23)$. Chen et al. found that HoxB3 promoted the progress of prostate cancer by up-regulating CDCA3 expression (24). In colorectal cancer, CDCA3 controlled G1/S phase transition by regulating the expression of transcription factor E2F1. Knockdown of CDCA3 expression led to stagnation of G1/S phase transition, which in turn led to inhibit tumor cell growth (26). The mechanism of CDCA3 in gastric cancer had not been thoroughly studied. Our data showed that CDCA3 was highly expressed in gastric cancer, and its high expression was associated with better prognosis-OS, FP, PPS - in patients with gastric cancer.

Cell division cycle-related protein 4 was a member of the TRIP-Br transcription co-factor family and a target gene of transcription factor E2F, which could inhibit the transcriptional activation and cell proliferation dependent on E2F $(5,27)$. CDCA4 was also found to promote the proliferation of breast cancer cells and reduce their apoptosis (28). Other studies had suggested that CDCA4 might be involved in the expression regulation of oncogene JUN (27). There were few studies on CDCA4 in other tumors. This data showed that the expression level of CDCA4 increased in gastric cancer, and it was worth noting that its high expression was usually associated with poor prognosis in patients with gastric cancer.

CDCA5 was a key regulator of sister chromatid aggregation and separation during cell division. CDCA5 played a role in multisystem malignancies and was seen as an indicator of poor prognosis. Studies showed that CDCA5 was upregulated in hepatocellular carcinoma (HCC), and increased expression of CDCA5 was associated with poor prognosis in HCC tissues (29). It was also suggested that the CDCA5 may enhance cell proliferation and inhibit apoptosis through the AKT pathway in HCC (30). In esophageal squamous cell carcinoma, increased CDCA5 expression was associated with advanced TNM staging and reduced overall survival (31). CDCA5 might also promote the progression of colorectal cancer by activating ERK signaling pathway (32). Meanwhile, CDCA5 expression increased in lung cancer, gastric cancer, bladder cancer, breast cancer, and melanoma, and might play a carcinogenic role by promoting cell proliferation and growth (33-37). Our data observed that CDCA5 was overexpressed in gastric cancer tissues compared with normal gastric tissues. Figure 5 showed that high expression of CDCA5 predicted better OS, FP, PPS in gastric cancer patients.

CDCA6, also known as CBX2 (chromobox2), encoded a component of the polycomb multiprotein complex, which might maintain the transcriptionally repressive state of many genes throughout development. Mechanistically, the overexpression of CBX2 might maintain cancer stem cells in an undifferentiated state and inhibit tumor suppressors, which in turn led to poor survival (38). Studies reported that CBX2 expression was upregulated in breast cancer, prostate cancer, osteosarcoma, hepatocellular carcinoma, and elevated CBX2 expression was significantly and independently associated with poor survival (39-42). In gastric cancer, CDCA6, namely CBX2, was overexpressed in GC tissues compared with adjacent normal samples. High expression of CDCA6 was associated with poor prognosis, namely, lower OS, FP, PPS.

CDCA7 expression was significantly elevated in gastric cancer. Ye et al. reported that high expression of CDCA7 was associated with poorer disease-free survival in patients with breast cancer (43). Currently, there were few studies on CDCA7 in gastric cancer. Our data suggested that overexpression of CDCA7 meant better OS, FP, PPS in GC patients.

CDCA8 encoded a crucial component of the chromosomal passenger complex, which acted as a key regulator of mitosis (37). CDCA8 overexpression was detected in various malignant tumors, such as breast cancer, bladder cancer, cutaneous melanoma, lung cancer, renal clear cell carcinoma $(6,44-47)$. Bi et al. demonstrated that the high expression level of CDCA8 was correlated with poor clinicopathological features of bladder cancer patients (6). Jiao et al. found that the increased expression of CDCA8 was positively associated with FOXM1 expression and shorter overall survival in breast cancer (47). Ci et al. discovered that overexpression of CDCA8 promoted the malignant progression of cutaneous melanoma and lead to poor prognosis (46). A study by Hayama et al. showed that overexpression of CDCA8 played a significant role in lung carcinogenesis (44). Our data indicated that CDCA8 is upregulated in gastric cancer. Meanwhile, overexpression of CDCA8 was correlated with better prognosis-OS, FP, PPS.

Abnormal expression of the CDCA protein family in a variety of tumors had been recognized. However, the study of CDCA protein subtypes in gastric cancer was still poorly understood. In this study, bioinformatics was used for the first time to study the expression pattern and prognostic value of CDCA family subtypes in gastric cancer. Our data showed that CDCA family members were highly expressed in gastric cancer. The results also suggested that CDCA4/6 
might be potential therapeutic targets for gastric cancer. And CDCA1/2/3/5/7/8 were expected to be new prognostic markers for gastric cancer. These results needed to be further confirmed by subsequent experiments. Although the research on CDCA protein family and gastric cancer was still in the early stage, its potential clinical value needed to be further studied.

\section{Acknowledgments}

We sincerely thank Professor Shokrollah Elahi for his help in polishing the manuscript.

Funding: None.

\section{Footnote}

Reporting Checklist: The authors have completed the MDAR checklist. Available at https://dx.doi.org/10.21037/ tcr-20-1050

Conflicts of Interest: All authors have completed the ICMJE uniform disclosure form (available at https://dx.doi. org/10.21037/tcr-20-1050). The authors have no conflicts of interest to declare.

Ethical Statement: The authors are accountable for all aspects of the work in ensuring that questions related to the accuracy or integrity of any part of the work are appropriately investigated and resolved. The study was conducted in accordance with the Declaration of Helsinki (as revised in 2013). Institutional ethical approval and informed consent were waived.

Open Access Statement: This is an Open Access article distributed in accordance with the Creative Commons Attribution-NonCommercial-NoDerivs 4.0 International License (CC BY-NC-ND 4.0), which permits the noncommercial replication and distribution of the article with the strict proviso that no changes or edits are made and the original work is properly cited (including links to both the formal publication through the relevant DOI and the license). See: https://creativecommons.org/licenses/by-nc-nd/4.0/.

\section{References}

1. GBD 2017 Stomach Cancer Collaborators. The global, regional, and national burden of stomach cancer in 195 countries, 1990-2017: a systematic analysis for the Global
Burden of Disease study 2017. Lancet Gastroenterol Hepatol 2020;5:42-54.

2. Thang PM, Takano A, Yoshitake Y, et al. Cell division cycle associated 1 as a novel prognostic biomarker and therapeutic target for oral cancer. Int J Oncol 2016;49:1385-93.

3. Harao M, Hirata S, Irie A, et al. HLA-A2-restricted CTL epitopes of a novel lung cancer-associated cancer testis antigen, cell division cycle associated 1, can induce tumorreactive CTL. Int J Cancer 2008;123:2616-25.

4. Kobayashi Y, Takano A, Miyagi Y, et al. Cell division cycle-associated protein 1 overexpression is essential for the malignant potential of colorectal cancers. Int J Oncol 2014;44:69-77.

5. Pang S, Xu Y, Chen J, et al. Knockdown of cell division cycle-associated protein 4 expression inhibits proliferation of triple negative breast cancer MDA-MB-231 cells in vitro and in vivo. Oncol Lett 2019;17:4393-400.

6. Bi Y, Chen S, Jiang J, et al. CDCA8 expression and its clinical relevance in patients with bladder cancer. Medicine (Baltimore) 2018;97:e11899.

7. Adams MN, Burgess JT, He Y, et al. Expression of CDCA3 Is a Prognostic Biomarker and Potential Therapeutic Target in Non-Small Cell Lung Cancer. J Thorac Oncol 2017;12:1071-84.

8. Zhang Y, Yin W, Cao W, et al. CDCA3 is a potential prognostic marker that promotes cell proliferation in gastric cancer. Oncol Rep 2019;41:2471-81.

9. Rhodes DR, Kalyana-Sundaram S, Mahavisno V, et al. Oncomine 3.0: genes, pathways, and networks in a collection of 18,000 cancer gene expression profiles. Neoplasia 2007;9:166-80.

10. Tang Z, Li C, Kang B, et al. GEPIA: a web server for cancer and normal gene expression profiling and interactive analyses. Nucleic Acids Res 2017;45:W98-102.

11. Nagy Á, Lánczky A, Menyhárt O, et al. Validation of miRNA prognostic power in hepatocellular carcinoma using expression data of independent datasets. Sci Rep 2018;8:9227.

12. Gao J, Aksoy BA, Dogrusoz U, et al. Integrative analysis of complex cancer genomics and clinical profiles using the cBioPortal. Sci Signal 2013;6:pl1.

13. Dennis G Jr, Sherman BT, Hosack DA, et al. DAVID: Database for Annotation, Visualization, and Integrated Discovery. Genome Biol 2003;4:P3.

14. Szklarczyk D, Morris JH, Cook H, et al. The STRING database in 2017: quality-controlled protein-protein association networks, made broadly accessible. Nucleic 
Acids Res 2017;45:D362-8.

15. Tokuzumi A, Fukushima S, Miyashita A, et al. Cell division cycle-associated protein 1 as a new melanoma-associated antigen. J Dermatol 2016;43:1399-405.

16. Ohnuma S, Miura K, Horii A, et al. Cancer-associated splicing variants of the CDCA1 and MSMB genes expressed in cancer cell lines and surgically resected gastric cancer tissues. Surgery 2009;145:57-68.

17. Zhao Z, Li C, Song B, et al. pH low insertion peptide mediated cell division cycle-associated protein 1 -siRNA transportation for prostatic cancer therapy targeted to the tumor microenvironment. Biochem Biophys Res Commun 2018;503:1761-7.

18. Obara W, Sato F, Takeda K, et al. Phase I clinical trial of cell division associated 1 (CDCA1) peptide vaccination for castration resistant prostate cancer. Cancer Sci 2017;108:1452-7.

19. Vagnarelli P. Repo-man at the intersection of chromatin remodelling, DNA repair, nuclear envelope organization, and cancer progression. Adv Exp Med Biol 2014;773:401-14.

20. Uchida F, Uzawa K, Kasamatsu A, et al. Overexpression of CDCA2 in human squamous cell carcinoma: correlation with prevention of $\mathrm{G} 1$ phase arrest and apoptosis. PLoS One 2013;8:e56381.

21. Shi R, Zhang C, Wu Y, et al. CDCA2 promotes lung adenocarcinoma cell proliferation and predicts poor survival in lung adenocarcinoma patients. Oncotarget 2017;8:19768-79.

22. Feng $Y$, Qian $W$, Zhang $Y$, et al. CDCA2 promotes the proliferation of colorectal cancer cells by activating the AKT/CCND1 pathway in vitro and in vivo. BMC Cancer 2019;19:576.

23. Phan NN, Wang CY, Li KL, et al. Distinct expression of CDCA3, CDCA5, and CDCA8 leads to shorter relapse free survival in breast cancer patient. Oncotarget 2018;9:6977-92.

24. Chen J, Zhu S, Jiang N, et al. HoxB3 promotes prostate cancer cell progression by transactivating CDCA3. Cancer Lett 2013;330:217-24.

25. Uchida F, Uzawa K, Kasamatsu A, et al. Overexpression of cell cycle regulator CDCA3 promotes oral cancer progression by enhancing cell proliferation with prevention of G1 phase arrest. BMC Cancer 2012;12:321.

26. Qian $W$, Zhang Z, Peng $W$, et al. CDCA3 mediates p21dependent proliferation by regulating E2F1 expression in colorectal cancer. Int J Oncol 2018;53:2021-33.

27. Tategu M, Nakagawa H, Hayashi R, et al. Transcriptional co-factor CDCA4 participates in the regulation of JUN oncogene expression. Biochimie 2008;90:1515-22.

28. Xu Y, Wu X, Li F, et al. CDCA4, a downstream gene of the Nrf2 signaling pathway, regulates cell proliferation and apoptosis in the MCF-7/ADM human breast cancer cell line. Mol Med Rep 2018;17:1507-12.

29. Tian Y, Wu J, Chagas C, et al. CDCA5 overexpression is an Indicator of poor prognosis in patients with hepatocellular carcinoma (HCC). BMC Cancer 2018;18:1187.

30. Chen H, Chen J, Zhao L, et al. CDCA5, Transcribed by E2F1, Promotes Oncogenesis by Enhancing Cell Proliferation and Inhibiting Apoptosis via the AKT Pathway in Hepatocellular Carcinoma. J Cancer 2019;10:1846-54.

31. Xu J, Zhu C, Yu Y, et al. Systematic cancer-testis gene expression analysis identified CDCA5 as a potential therapeutic target in esophageal squamous cell carcinoma. EBioMedicine 2019;46:54-65.

32. Shen A, Liu L, Chen H, et al. Cell division cycle associated 5 promotes colorectal cancer progression by activating the ERK signaling pathway. Oncogenesis 2019;8:19.

33. Zhang Z, Shen M, Zhou G. Upregulation of CDCA5 promotes gastric cancer malignant progression via influencing cyclin E1. Biochem Biophys Res Commun 2018;496:482-9.

34. Zhou Q, Ren J, Hou J, et al. Co-expression network analysis identified candidate biomarkers in association with progression and prognosis of breast cancer. J Cancer Res Clin Oncol 2019;145:2383-96.

35. Xu T, Ma M, Dai J, et al. Gene expression screening identifies CDCA5 as a potential therapeutic target in acral melanoma. Hum Pathol 2018;75:137-45.

36. Nguyen MH, Koinuma J, Ueda K, et al. Phosphorylation and activation of cell division cycle associated 5 by mitogen-activated protein kinase play a crucial role in human lung carcinogenesis. Cancer Res 2010;70:5337-47.

37. Chang IW, Lin VC, He HL, et al. CDCA5 overexpression is an indicator of poor prognosis in patients with urothelial carcinomas of the upper urinary tract and urinary bladder. Am J Transl Res 2015;7:710-22.

38. Jangal M, Lebeau B, Witcher M. Beyond EZH2: is the polycomb protein $\mathrm{CBX} 2$ an emerging target for anticancer therapy. Expert Opin Ther Targets 2019;23:565-78.

39. Zheng S, Lv P, Su J, et al. Overexpression of CBX2 in breast cancer promotes tumor progression through the PI3K/AKT signaling pathway. Am J Transl Res 2019;11:1668-82. 
40. Li J, Guo ZX, Chen JA, et al. Studies on the role of chromobox protein homolog 2 in the inhibition of progression of hepatoma. Zhonghua Gan Zang Bing Za Zhi 2019;27:192-7.

41. Han Q, Li C, Cao Y, et al. CBX2 is a functional target of miRNA let-7a and acts as a tumor promoter in osteosarcoma. Cancer Med 2019;8:3981-91.

42. Clermont PL, Crea F, Chiang YT, et al. Identification of the epigenetic reader $\mathrm{CBX} 2$ as a potential drug target in advanced prostate cancer. Clin Epigenetics 2016;8:16.

43. Ye L, Li F, Song Y, et al. Overexpression of CDCA7 predicts poor prognosis and induces EZH2-mediated progression of triple-negative breast cancer. Int J Cancer 2018;143:2602-13.

44. Hayama S, Daigo Y, Yamabuki T, et al. Phosphorylation

Cite this article as: $\mathrm{Li}$ Z, Liu Z, Li C, Liu Q, Tan B, Liu Y, Zhang Y, Li Y. CDCA1/2/3/5/7/8 as novel prognostic biomarkers and CDCA $4 / 6$ as potential targets for gastric cancer. Transl Cancer Res 2021;10(7):3404-3417. doi: 10.21037/tcr-201050 and activation of cell division cycle associated 8 by aurora kinase B plays a significant role in human lung carcinogenesis. Cancer Res 2007;67:4113-22.

45. Gu Y, Lu L, Wu L, et al. Identification of prognostic genes in kidney renal clear cell carcinoma by RNA-seq data analysis. Mol Med Rep 2017;15:1661-7.

46. Ci C, Tang B, Lyu D, et al. Overexpression of CDCA8 promotes the malignant progression of cutaneous melanoma and leads to poor prognosis. Int J Mol Med 2019;43:404-12.

47. Jiao DC, Lu ZD, Qiao JH, et al. Expression of CDCA8 correlates closely with FOXM1 in breast cancer: public microarray data analysis and immunohistochemical study. Neoplasma 2015;62:464-9. 